\title{
The Impact of Inpatient Endoscopy in the Management of Anticoagulant-Associated Non- Variceal Upper Gastrointestinal Bleed
}

\author{
Abdallah Abdallah ( $\nabla$ abed8k@gmail.com ) \\ Magnolia Regional Health Center https://orcid.org/0000-0002-5452-8607 \\ Christopher Smith \\ Magnolia Regional Health Center
}

Hayes Baker

Magnolia Regional Health Center

Chris Tucker

Magnolia Regional Health Center

Todd Coffey

Idaho College of Osteopathy: Idaho College of Osteopathic Medicine

Dantwan Smith

Magnolia Regional Health Center

Kaycee Burcham

Magnolia Regional Health Center

Waqaar Arshad

Magnolia Regional Health Center

Raul Ramirez

Magnolia Regional Health Center

Brandon Moore

Magnolia Regional Health Center

Adriano Duran

Magnolia Regional Health Center

Neil Shah

UNC-Chapel Hill: The University of North Carolina at Chapel Hill

\section{Research Article}

Keywords: Non-variceal upper GIB, endoscopy and GIB on anticoagulant, anticoagulant associated GIB

Posted Date: February 24th, 2021

DOl: https://doi.org/10.21203/rs.3.rs-264854/v1 
License: (c) (i) This work is licensed under a Creative Commons Attribution 4.0 International License. Read Full License 


\section{Abstract}

Background: Patients on systemic anticoagulation are at increased risk for gastrointestinal bleeding (GIB). Guidelines recommend endoscopy within 24 hours for diagnostic and therapeutic purposes. Unfortunately, lack of resources and/or gastroenterologists in small, community centers limit the ability to perform timely endoscopy. Conservative management is used in this setting with delayed or outpatient endoscopy, specifically in those who respond to medical therapy. There is minimal data on the outcomes of patients managed in this fashion.

Objective: To compare outcomes of patients on systemic anticoagulation who present with non-variceal upper GIB in a small community hospital.

Design: We conducted a retrospective cohort chart review.

Participants: 115 adult patients who met the inclusion criteria for non-variceal upper GIB on systemic anticoagulation. Excluded patients were those on dual antiplatelet therapy, history of decompensated cirrhosis, variceal bleeds, active GIB identified on imaging (defined as contrast extravasation on CT angiogram or positive tagged RBC nuclear scan), and those with gastrointestinal malignancy.

Interventions: Resuscitative medical therapy (MT) alone vs inpatient endoscopy with resuscitative MT.

Main Measures: Outcomes included red blood cell (RBC) transfusions, re-admission for GIB, and 30-day all-cause mortality. Sub-group analysis was also performed based on endoscopy timeline and anticoagulation type.

Key Results: Patient in MT group required less RBC transfusions compared to endoscopy group (1.4 vs 2.5 average units, [95\% Cl 1.01-1.87]; $P=0.004$ ). There was no statistical significance for re-admission for gastrointestinal bleeding or mortality between both groups. Moreover, there was no significant difference in RBC transfusions based on EGD timing. Most patients were on warfarin $(n=47)$ and there were no differences in re-admission for GIB or mortality based on anticoagulant use.

Conclusion: Our study suggests that there is no significant therapeutic benefits to inpatient endoscopy in comparison to medical management alone in anticoagulated patients who present with non-variceal UGIB.

\section{Introduction}

Upper gastrointestinal bleeding (UGIB) is defined as bleeding located proximal to the ligament of Treitz. Incidence is approximately $50-150$ per 10000 patients yearly ${ }^{[1]}$, with significant mortality in hospitalized patients (3.5-10\%) ${ }^{[5]}$. Most UGIB is related to peptic ulcer disease (up to $50 \%$ ), followed by gastroduodenal erosions (up to $15 \%$ ) ${ }^{[5]}$. Gastroduodenal erosions and direct mucosal injury are the hypothesized GIB source related to anticoagulant use. With the increase of anticoagulant use, UGIB incidence is expected to rise challenging the current conventional approach in management of UGIB. 
Hospitalization for UGIB requires a careful approach that relies on initial supportive management, use of blood products or reversal agents, and timing for endoscopy. The current recommendation on initial management relies on hemodynamic stabilization, placement of two large bore (18 gauge or more), followed by determining patient's risk for re-bleeding and mortality ${ }^{[2]}$. Glasgow-Blatchford bleeding score, AIMS-65 score, and the Clinical Rockall score are methods used to risk stratify patient and aid in the decision for the need for early endoscopic intervention, re-bleeding risk, and prediction of in-hospital

mortality ${ }^{[2][4]}$. However, these scoring systems do not factor in the use of anticoagulation. As a result, much of the decision on timing of endoscopy relies on the response to initial medical management and the clinical judgement of an experienced gastroenterologist.

Regardless of the subtypes of UGIB or severity, early endoscopic intervention ( $<24$ hours of presentation) remains the standard recommendation according to most guidelines ${ }^{[2]}$. Sparse amount of literature is available on the role of endoscopy related to UGIB in the setting of anticoagulant use. For that reason, we decided to study UGIB in the particular patient population who are on anticoagulation and evaluate whether endoscopy, its timeframe, and other potential prognostic factors influenced re-bleeding rate or inhospital mortality.

\section{Methods}

We conducted a 2-year retrospective cohort study involving chart review of 323 patients admitted for acute blood loss anemia and GIB.

Of those, 115 adult patients met the selection criteria including patients on chronic anticoagulation with or without single antiplatelet therapy that were admitted for non-variceal UGIB. We excluded patients on dual antiplatelet therapy, history of cirrhosis, variceal bleeds, active GIB identified on imaging (defined as contrast extravasation on CT or positive tagged RBC nuclear scan), and gastrointestinal malignancy. The patients underwent either medical therapy (MT) with endoscopy or MT alone prior to discharge.

Esophagogastroduodenoscopy (EGD) were carried out at different times throughout hospital stay (within $24 \mathrm{hr}, 48 \mathrm{hr}, 72 \mathrm{hr}$, and above $72 \mathrm{hr}$ after admission) and outcomes were recorded for each group. Early EGD was considered within 24hours. Medical management included intravenous (IV) crystalloid fluid resuscitation, blood product transfusion (packed red blood cells, fresh frozen plasma, platelets, cryoprecipitate), and vitamin K, Reversal agents (Idarucizumab, prothrombin complex concentrate) were used in select patients who were using dabigatran or warfarin. Acid suppression included IV pantoprazole-80mg bolus followed by continuous infusion. The primary endpoints were re-admission for GIB, number of red blood cell (RBC) transfusions, and 30 day all-cause mortality. We also analyzed readmission for GIB and mortality based on the timeframe of endoscopy and anticoagulant used. Subgroup analysis of the endpoints were done based on the timing of endoscopy and specific anticoagulant agent. To eliminate endoscopy operator findings or interventional bias, all groups were managed by a single gastroenterologist. Association between primary endpoints and potential prognostic factors was evaluated using a Chi-square test of association for categorical variables and logistic regression for continuous variables. When the contingency table cells were small, Fisher's exact test was used. Mean 
differences between multiple groupings were analyzed using analysis of variance, with a logarithmic transformation when necessary to ensure homogeneity of variance.

\section{Results}

Out of 115 patients, 61 underwent esophagogastroduodenoscopy (EGD) and 54 received MT only. The patients were mostly Caucasian and there were no significant differences in age, average hospital stay, baseline hemoglobin, or co-morbidities between both groups (Table 1). The EGD group was divided based on time/days to procedure: $<24$ hours (10), $24-48$ hours (32), 48-72 hours (10), $>72$ hours (9) as in Table 3. Bleeding etiology was discovered in $100 \%$ of the EGD group, but hemostatic intervention was required in only 2 patients. Neither of these had re-bleed or mortality in 30 days. RBC transfusions were adjusted to average admission hemoglobin for each group. In those with MT alone, 1.4 units of RBC were transfused on average compared to 2.5 units in the EGD + MT group, [95\% $\mathrm{Cl} 1.01-1.87] ; \mathrm{P}=0.004$ (Table 2). There was no significant difference in RBC transfusions based on EGD timing. A total of 5 patients were re-admitted for GIB, 3 in the EGD group, and 2 in the MT group as in Table $3(p=0.20)$. Readmissions from the MT group had EGD which discovered a bleeding source. There were 3 deaths, one in the EGD group ( $<24$ hours) and 2 in the MT group ( $p=0.60)$. Of the MT group, one patient died of cardiac arrest from hemorrhagic shock within 24 hours. The other two died of cardiopulmonary arrest as a result of heart failure. Most patients were on warfarin $(n=47)$ and there were no differences in re-admission for GIB or mortality based on anticoagulant use (Table 4). 
Table 1

Demographic data comparing endoscopy group with Medical Therapy only group.

\begin{tabular}{|c|c|c|}
\hline & Endoscopy Group $(n=61)$ & Medical Therapy $(n=54)$ \\
\hline Average Age (yrs) & 75.1 & 74.8 \\
\hline Ethnicity (\%) & $97(59)$ & $94(51)$ \\
\hline Caucasian & $3(2)$ & $6(3)$ \\
\hline \multicolumn{3}{|l|}{ African American } \\
\hline Male Gender (\%) & $47(29)$ & $33(18)$ \\
\hline Average Hemoglobin (g/dL) & 8.89 & 9.23 \\
\hline Average Hospital LOS (days) & 6.50 & 5.98 \\
\hline Etiology of GIB (\%) & $48(29)$ & $\mathrm{N} / \mathrm{A}$ \\
\hline Gastroduodenitis & $29(18)$ & \\
\hline Peptic Ulcer Disease & $20(12)$ & \\
\hline Erosions & $3.3(2)$ & \\
\hline \multicolumn{3}{|l|}{ Mallory-Weiss Tear } \\
\hline Anticoagulant (\%) & $31(19)$ & $52(28)$ \\
\hline Warfarin & $31(19)$ & $39(21)$ \\
\hline Apixaban & $5(3)$ & $9.3(5)$ \\
\hline Dabigatran & $20(12)$ & $13(7)$ \\
\hline Rivaroxaban & $1.6(1)$ & 0 \\
\hline \multicolumn{3}{|l|}{ Enoxaparin } \\
\hline Antiplatelet (\%) & $51(31)$ & $63(34)$ \\
\hline Aspirin & $29(18)$ & $46(25)$ \\
\hline Clopidogrel & $8.2(5)$ & $3.7(2)$ \\
\hline \multicolumn{3}{|l|}{ None } \\
\hline Reversal agent (\%) & $13(8)$ & $13(7)$ \\
\hline Vitamin $\mathrm{K}$ & $1.6(1)$ & 0 \\
\hline PCC & & \\
\hline
\end{tabular}


Table 2

Mean (SD) Number of Packed Red Blood Cell Transfusions by Endoscopy.

\begin{tabular}{|llll|}
\hline Grouping & N & Mean & Standard Deviation (SD) \\
\hline No Endoscopy & 54 & 1.44 & 1.61 \\
\hline Endoscopy Performed & 61 & 2.49 & 2.53 \\
\hline
\end{tabular}

Table 3

Comparing endoscopy timing with 30 day UGIB related re-admission (re-bleed) and mortality.

\begin{tabular}{|llll|}
\hline Timing of Endoscopy (HR) & Number of Cases (\%) & Re-bleed (\%) & Mortality (\%) \\
\hline$<24 \mathrm{HR}$ & $10(8.7 \%)$ & $1(20 \%)$ & $1(33.3 \%)$ \\
\hline $24-48 \mathrm{HR}$ & $32(27.8 \%)$ & $1(20 \%)$ & 0 \\
\hline $48-72 \mathrm{HR}$ & $10(8.7 \%)$ & 0 & 0 \\
\hline$>72 \mathrm{HR}$ & $9(7.8 \%)$ & 0 & 0 \\
\hline No Endoscopy & $54(47.0 \%)$ & $3(60 \%)$ & $2(66.7 \%)$ \\
\hline Total & 115 & 5 & 3 \\
\hline
\end{tabular}

Table 4

Comparing anticoagulant with re-bleed (30 GIB related re-admission) and 30-day mortality.

\begin{tabular}{|llll|}
\hline Name of anticoagulant & Number of Cases (\%) & Re-bleed (\%) & Mortality (\%) \\
\hline Warfarin & $47(40.9 \%)$ & $1(20 \%)$ & $1(33.3 \%)$ \\
\hline Apixaban & $40(34.8 \%)$ & $1(20 \%)$ & $2(66.7 \%)$ \\
\hline Rivaroxaban & $19(16.5 \%)$ & $2(40 \%)$ & 0 \\
\hline Dabigatran & $8(7.0 \%)$ & $1(20 \%)$ & 0 \\
\hline Enoxaparin & $1(0.9 \%)$ & 0 & 0 \\
\hline Total & 115 & 5 & 3 \\
\hline
\end{tabular}

\section{Discussion}

Our goal was to look at outcomes in patients with non-variceal UGIB at a small, community medical center where urgent EGD cannot always be performed. We hypothesized that the anticoagulated subset of patients presenting with non-variceal and non-active GIB can be safely managed by supportive medical therapy and delayed endoscopy. In this retrospective cohort study, we found that patients that received MT only had significantly fewer blood transfusions compared to those undergoing endoscopy. 
GIB re-admission and mortality were not statistically different in both groups. The higher transfusion requirement in the EGD group suggests that these patients were refractory to medical therapy and may carry higher bleeding stigmata necessitating therapeutic maneuver. However, only two patients required a therapeutic maneuver in the endoscopy group while all others were only diagnostic. Moreover, there was no report of endoscopy associated bleeding as a complication in the studied group. When analyzing by anticoagulants, Rivaroxaban had the most re-admissions for GIB (2 of 5), but not statistically significant compared to other agents. Our study had multiple limitations. First, the selected population was small, retrospective and not-randomized to intervention. Furthermore, long term outcomes (more than 30 days) were not assessed due to lack of outpatient data and loss of follow up of patients. RBC transfusion requirement could potentially be confounded by hemodilution, volume status, and hemodynamics. The population was specifically selected for the anticoagulated patient which may not be generalizable to all patients presenting with UGIB. But our study does suggest that delayed endoscopy for these patients may not significantly alter outcomes such as recurrent GIB and mortality. Other studies have demonstrated that early endoscopy within 24 hours also did not improve re-bleeding, hospitalization duration, or mortality, and were predictor of worse outcomes ${ }^{[3][5]}$. Similarly, our study suggests that in a small, community center, there may be no added benefit for inpatient endoscopy in the management of nonvariceal UGIB specifically in anticoagulated patients. Nevertheless, a randomized, large sample study is needed to substantiate this claim.

\section{Declarations}

\section{Contributorship statement}

We sincerely thank Dr. Hayes Baker, Dr. Neil Shah, Dr. Tucker, and Dr. Todd Coffey who acted as scientific advisor and for the support in the analysis, interpretation, and manuscript editing. A.A. and S.C. conceived the presented hypothesis of the study. A.A. wrote the manuscript. C.T. carried statistical computation. A.A. S.C. S.D. B.K. A.W. K.R. O.Z. K.L. R.R. M.B. D.A. assisted with data collection.

\section{Competing interests}

None

\section{Funding}

None

\section{Data sharing statement}

All data requests should be submitted to the corresponding author for consideration.

\section{IRB Approval}

Date of approval December 11, 2018. IRB\# 2018-652 under Nova Southeastern University IRB 


\section{References}

1. -Palmer KR. Non-variceal upper gastrointestinal haemorrhage: guidelines. Gut 2002. https://gut.bmj.com/content/51/suppl_4/iv1 (accessed April 16, 2020).

2. -Barkun, A., Almadi, M., Kuipers, E., Laine, L., Sung, J., Tse, F., Leontiadis, G., Abraham, N., Calvet, X., Chan, F., Douketis, J., Enns, R., Gralnek, I., Jairath, V., Jensen, D., Lau, J., Lip, G., Loffroy, R., MalufFilho, F., Meltzer, A., Reddy, N., Saltzman, J., Marshall, J. and Bardou, M., 2020. Management Of Nonvariceal Upper Gastrointestinal Bleeding: Guideline Recommendations From The International Consensus Group. [online] Annals of Internal Medicine. Available at: $<$ https://annals.org/aim/fullarticle/2753604/management-nonvariceal-upper-gastrointestinalbleeding-guideline-recommendations-from-international-consensus> [Accessed 2 April 2020].Kim, Jiwon. "Management and Prevention of Upper GI Bleed." Management and Prevention of Upper GI Bleed, 2011, .

3. -Kumar NL, Cohen AJ, Nayor J, Claggett BL, Saltzman JR. Timing of upper endoscopy influences outcomes in patients with acute nonvariceal upper GI bleeding. Gastrointest Endosc. 2017;85:945952.e1. https://www.ncbi.nlm.nih.gov/pubmed/27693643

4. -Hwang, J. (2012). The role of endoscopy in the management of acute non-variceal upper GI bleeding. Retrieved from https://www.giejournal.org/article/S0016-5107(12)00198-8/fulltext Gastrointest Endosc 2012;75:1132-1138 / DOI: http://dx.doi.org/10.1016/j.gie.2012.02.033

5. -Vaduganathan, M. and Bhatt, D., 2020. Gastrointestinal Bleeding With Oral Anticoagulation: Understanding The Scope Of The Problem. [online] Clinical Gastroenterology and Hepatology. Available at: <https://www.cghjournal.org/article/S1542-3565(17)30018-6/fulltext> [Accessed 2 April 2020].

6. -Lau, James Y.W., et al. "Timing of Endoscopy for Acute Upper Gastrointestinal Bleeding: NEJM." New England Journal aof Medicine, 2 Apr. 2020, .

\section{Figures}




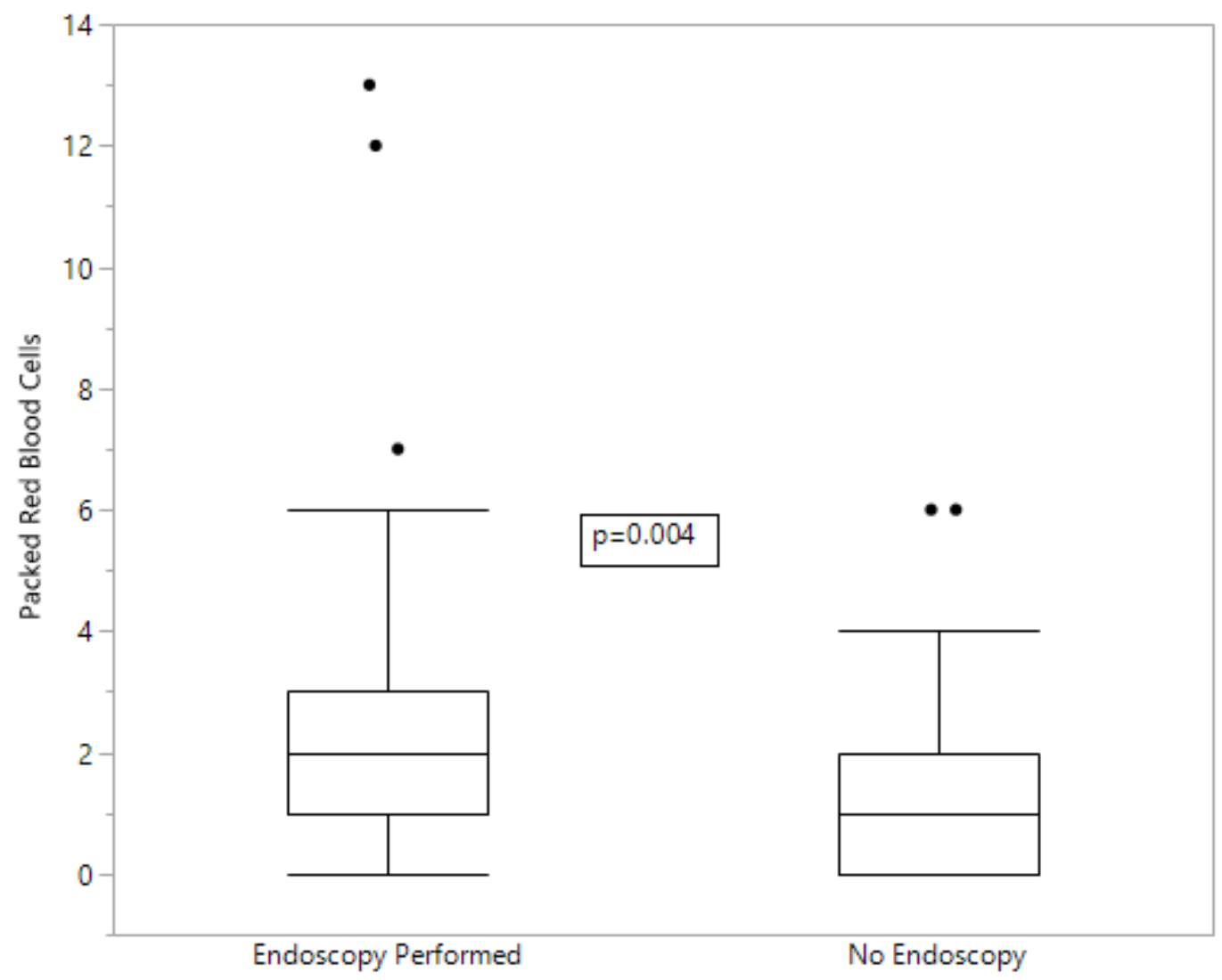

Figure 1

Histograms for Number of Average Packed Red Blood Cell Transfusions by Endoscopy 